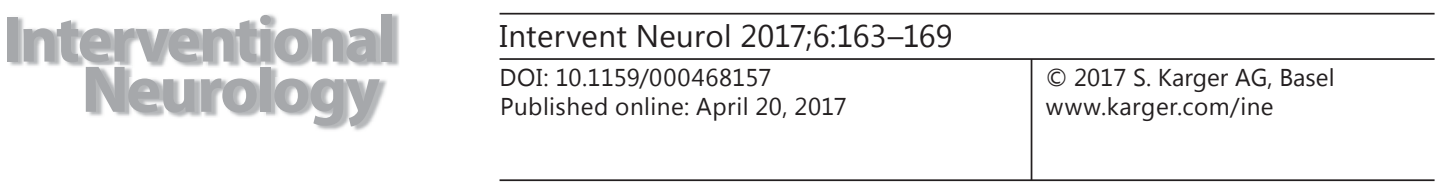

\title{
Feasibility of Real-Time Angiographic Perfusion Imaging in the Treatment of Cerebral Vasospasm
}

\author{
Christopher Donaldson $^{a} \quad$ Anthea H. O'Neill ${ }^{b}$ Lee-Anne Slater ${ }^{b}$ c \\ Winston Chong ${ }^{b, c}$ Leon T. Lai ${ }^{a, d}$ Ronil V. Chandrab, \\ ${ }^{a}$ Department of Neurosurgery, Monash Health, ${ }^{b}$ Faculty of Medicine, Nursing and Health \\ Sciences, Monash University, ${ }^{C}$ Diagnostic and Interventional Neuroradiology, Monash \\ Imaging, Monash Health, and d Department of Surgery, School of Clinical Sciences, Monash \\ University, Melbourne, VIC, Australia
}

\section{Keywords}

2-D perfusion - Perfusion analysis · Subarachnoid hemorrhage $\cdot$ Vasospasm - Vasospasm treatment

\begin{abstract}
Background: Objective assessment and quantification of the severity of cerebral vasospasm following aneurysmal subarachnoid hemorrhage is not routinely utilized. We investigated the feasibility of angiographic perfusion imaging derived from digital subtraction angiography (DSA) following endovascular vasospasm treatment procedures. Methodology: Real-time blood flow analysis was performed using parametric color coding on pre- and postintervention DSA. Semiquantitative parenchymal perfusion parameters (arrival time [AT] of contrast, time to peak [TTP] opacification, and mean transit time [MTT] of contrast) were calculated across 3 vascular territories (anterior cerebral artery $[A C A]$, middle cerebral artery $[\mathrm{MCA}]$, and lenticulostriate arteries) using standard 2-D angiographic perfusion software. The pre- and postintervention arterial vessel diameters were compared. Results: Twelve endovascular vasospasm treatments in 6 patients were performed. All patients received intra-arterial vasodilator therapy with either nimodipine, milrinone, or both. Following intra-arterial intervention, parenchymal flow analysis showed improvement in TTP and MTT across all vascular territories $(p<0.002)$ and improvement in AT in the ACA and MCA territories $(p<0.03)$. Improvement in parenchymal perfusion parameters was associated with improvement in vessel diameters in all territories following treatment $(p<0.05)$. Conclusion: Real-time parenchymal perfusion imaging during endovascular vasospasm treatment procedures is feasible and provides reliable semiquantitative measurement of angiographic treatment response.
\end{abstract}




\section{Introduction}

Delayed ischemic neurological injury from cerebral vasospasm remains a significant contributor to hospital-associated morbidity and mortality following aneurysmal subarachnoid hemorrhage [1-3]. The pathogenesis has been postulated to be multifactorial and includes reduced blood flow from angiographic vasospasm, microcirculatory constriction, microthrombosis, cortical spreading ischemia, and delayed effects of early brain injury [3-5]. Since the introduction of intra-arterial vasodilator agents and balloon angioplasty as potential treatment options for patients with cerebral vasospasm, few advances have been made in the quantification of vasospasm severity and objective angiographic assessment of treatment response. The current standard remains the comparative visual inspection of vessel diameter, parenchymal opacification, and cerebral flow using digital subtraction angiography (DSA) before and after intervention $[3,5]$. Whilst this provides a subjective measure of the macrovascular response, it may not reliably reflect the microvascular changes that are suspected to underpin clinical vasospasm $[4,6]$.

Recent introductions to software enabling 2-D perfusion analysis using parametric color coding have only had preliminary research into their value as a clinical tool, particularly in the assessment of the efficacy of vasospasm treatment [6-9]. This software produces a single image that uses parametric color coding to show the time course of density of contrast throughout a DSA series and enables on-table analysis of the efficacy of vasospasm treatment in both the micro- and macrovasculature. Confirmation of the feasibility and reliability of this technique as a means of quantifying radiographic response to vasospasm treatment is important in order to move towards assessment of clinical relevance. To this end, we performed a retrospective study to investigate the utility of 2-D perfusion imaging as a potential semiquantitative, objective measure of cerebral perfusion following treatment of cerebral vasospasm.

\section{Materials and Methods}

Inclusion and Exclusion Criteria

An institutional analysis was performed retrospectively on patients with aneurysmal subarachnoid hemorrhage who had their aneurysm treated within $24 \mathrm{~h}$ of presentation. Patients with a delayed acute neurological decline and a diagnosis of anterior circulation cerebral vasospasm confirmed on DSA were included in the study. Patients receiving balloon angioplasty were excluded. Consecutive patients that presented to the institution and satisfied inclusion criteria were enrolled over a 6-month time period.

\section{Digital Subtraction Angiography}

Cerebral angiography was performed on the Phillips AlluraClarity ${ }^{\mathrm{TM}}$ biplane flat detector system with ClarityIQ technology (Philips Medical Systems B.V., The Netherlands). We used standard cerebral angiography methods in all patients with transfemoral access and 5-Fr angiography catheters with a standard inner diameter of 0.038 inches. These were placed in the distal extracranial internal carotid artery, typically at the level of C2. Vasodilator treatments were administered as an intra-arterial infusion through the diagnostic angiography catheter. Vasodilating agents used included intra-arterial nimodipine, milrinone, or a combination of both, with dosage varying depending on the operator. Nimodipine was given at dosages of 2-5 mg and milrinone at between 7.5 and $10 \mathrm{mg}$, typically over 30-60 min. During the initial diagnostic runs and posttreatment runs, the catheter remained in an unchanged position.

\section{2-D Perfusion Software}

The Phillips 2-D perfusion software (Philips Medical Systems B.V.) was initiated on an independent workstation (XtraVision, Philips Healthcare, The Netherlands), and 2-D perfusion imaging was performed both before and after intervention. Angiographic runs for each set of 2-D perfusion data were acquired at a standardized contrast injection rate $(6 \mathrm{~mL}$ of contrast over $4 \mathrm{~mL} / \mathrm{s}$, with $1 \mathrm{~s}$ rate rise). The time of recording 


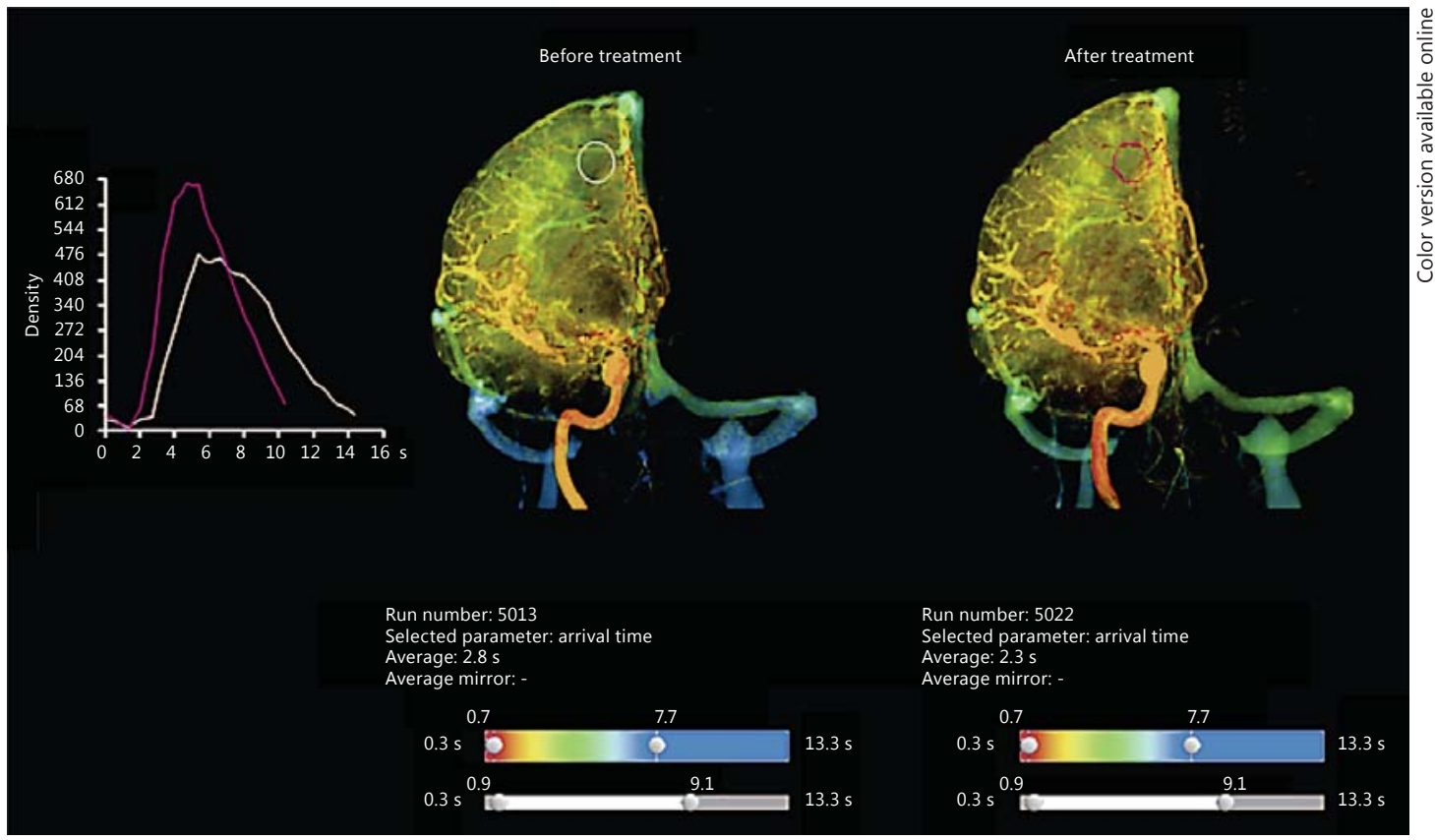

Fig. 1. 2-D perfusion parametric color-coded map of a patient undergoing right internal carotid artery vasodilator treatment with region of interest circle in the anterior cerebral artery territory, with arrival time of contrast as selected output parameter.

was determined on a patient-by-patient basis, and recording was continued until opacification of major venous sinuses. Time-density curves were automatically calculated from the DSA images with parametric color coding allowing postprocedural data extraction.

\section{Image Analysis}

Each patient had 4 angiographic images taken for analysis: 1 preintervention DSA image, 1 preintervention 2-D perfusion map, 1 postintervention DSA image, and 1 postintervention 2-D perfusion map. We measured vessel diameter on pre- and postintervention DSA images for each patient. These data were recorded in a de-identified manner. The pre- and postintervention 2-D perfusion maps were analyzed for 3 separate parameters that were automatically derived from the parametric color coding sequences: arrival time (AT) of contrast, mean transit time (MTT) of contrast, and the time to peak (TTP) opacification of contrast. Using a region of interest (ROI) circle placed in 3 separate vascular territories (anterior cerebral artery [ACA], middle cerebral artery [MCA], and lenticulostriate arteries [LtA]), an average value for each parameter in the ROI was calculated by the software (Fig. 1). Each territory was analyzed with 2 ROIs, and an average value was obtained across both. Large vessels were avoided when choosing the placement of the ROI to gain a more accurate representation of parenchymal tissue perfusion.

The percentage change in vessel diameter was assessed between the pre- and postintervention DSA images. The vessels measured correlated with the vascular territories measured by the 2-D perfusion software. The smallest-caliber segment of the proximal MCA (M1 segment) and the LtA were measured using the anteroposterior views of the angiogram. The smallest ACA (A2) segments were measured using the lateral view. The corresponding segment on the postintervention DSA was then measured with the same view and recorded in a de-identified manner.

\section{Statistical Analysis}

Statistical analysis was performed using Statistical Package for the Social Sciences (SPSS) software version 21 (SPSS Inc., Chicago, IL, USA). The pre- and postintervention cerebral DSA values were assessed using a 2 -sided Student paired $t$ test to test for a significant difference. The same statistical analysis was then performed on each 2-D perfusion parameter individually to assess for differences in pre- and postintervention values. Statistical significance was set to a probability value of $<0.05$. 
Table 1. General demographics

Donaldson et al.: Feasibility of Real-Time Angiographic Perfusion Imaging in the Treatment of Cerebral Vasospasm

$\begin{array}{lc}\text { Patients, } n & 6 \\ \text { Female, } n(\%) & 5(83.3) \\ \text { Mean age (range), years } & 52.8(38-72) \\ \text { Ruptured aneurysm location, } n & \\ \quad \text { PCoA } & 2 \\ \quad \text { ACoA } & 2 \\ \quad \text { MCA } & 2 \\ \text { Side of vasospasm, } n(\%) & 7(58.3) \\ \quad \text { Left } & 3(25.0) \\ \quad \text { Right } & 2(16.7) \\ \quad \text { Bilateral } & 12 \\ \text { Treatment episodes, } n & 2(1-5) \\ \text { Average treatment per patient (range), } n & \end{array}$

PCoA, posterior communicating artery; ACoA, anterior communicating artery; MCA, middle cerebral artery.

Table 2. Comparative analysis of 2-D perfusion parameters before and after vasospasm treatment

\begin{tabular}{llll}
\hline & $\begin{array}{l}\text { Mean preintervention } \\
\text { time, s }\end{array}$ & $\begin{array}{l}\text { Mean postintervention } \\
\text { time, s }\end{array}$ & $p$ value \\
\hline $\begin{array}{l}\text { Arrival time of contrast } \\
\text { ACA }\end{array}$ & 2.3 & 1.9 & $<0.03$ \\
MCA & 2.4 & 1.8 & $<0.002$ \\
LtA & 1.7 & 1.6 & $<0.15$ \\
\hline Mean transit time of contrast & & & $<0.004$ \\
ACA & 4.3 & 3.4 & $<0.003$ \\
MCA & 4.3 & 3.1 & $<0.015$ \\
LtA & 3.9 & 3.3 & $<0.004$ \\
Time to peak opacification of contrast & 3.8 & 3.1 & $<0.004$ \\
ACA & 3.8 & 2.9 & $<0.02$ \\
MCA & 3.4 & 2.9 & \\
LtA & & &
\end{tabular}

ACA, anterior cerebral artery; MCA, middle cerebral artery; LtA, lenticulostriate arteries.

\section{Results}

A total of 12 treatment episodes in 6 patients were included in the study. Table 1 summarizes the demographic data. The mean patient age was 52.8 years (range 38-72). Five patients were female. Four patients had only 1 treatment episode, 1 patient had 3 interventions, and 1 patient had 5 treatment episodes. The ruptured aneurysm causing subarachnoid hemorrhage was located on the posterior communicating artery in 2 patients, the anterior communicating artery in 2 patients, and the MCA in 2 patients. The vascular territory involved in vasospasm was bilateral in 2 patients. Both nimodipine and milrinone were administered in 10 of the 12 treatment episodes with nimodipine alone utilized for 1 treatment episode and milrinone alone used for 1 treatment episode.

Following endovascular treatment, mean angiographic vessel diameters significantly increased across all 3 vascular territories. The ACA vessels increased in diameter by $50.7 \%$ 
$(p<0.05)$, the MCA vessels by $85.8 \%(p<0.01)$, and the LtA vessels by $19.7 \%(p<0.01)$. This correlated well with the semiquantitative parameters in which parenchymal flow analysis showed improvement in TTP and MTT across all vascular territories $(p<0.002)$ and improvement in AT in the ACA and MCA territories $(p<0.03)$ (Table 2). Improvement in parenchymal perfusion parameters was associated with improvement in vessel diameters in all territories following treatment $(p<0.05)$.

\section{Discussion}

In the current study, semiquantitative measurements of parenchymal perfusion, with TTP and MTT, improved the following intra-arterial treatment for vasospasm across ACA, MCA, and LtA territories. This is important as previous studies investigating the use of computed tomography (CT) perfusion in the setting of vasospasm have highlighted that MTT and TTP are sensitive and early predictors of secondary cerebral infarction [10-12]. The results underscore the feasibility of such a technique in providing a real-time, semiquantitative assessment of cerebral micro- and macrovascular blood flow, which can allow for the quantification of vasospasm severity and objective angiographic assessment of treatment response.

There have been 2 previous articles that have discussed the use of 2-D perfusion imaging to assess radiographic response to vasospasm treatment. In 2014, Levitt et al. [6] analyzed the results of 2-D perfusion imaging using the Philips Allura workstation in 8 vascular territories across 4 patients and found that improvement was seen to varying degrees in parameters such as MTT (between -13 and 6\%) and TTP (between -31 and 38\%) across most treated vascular territories in individual patients. These results are less consistent than our findings, which show a significant improvement in MTT and TTP across all vascular territories. Nonetheless, it should be noted that Levitt et al. [6] utilized a different vasodilator agent, nicardipine, and had a smaller sample size. This may be one possible reason for the discrepancy in the results of the 2 studies.

Recently, Gölitz et al. [13] utilized the Siemens Leonardo workstation and iFlow software to analyze the angiographic response to intra-arterial nimodipine treatment for vasospasm in 17 patients. They found significant improvements in cerebral circulation time after treatment, similar to our improvement in MTT. Gölitz et al. [13] also assessed TTP contrast concentration in each vessel, which was not significantly different after intervention. In contrast, we measured TTP of the parenchymal contrast blush, which better assesses the microcirculation and was significantly improved after intervention. A direct comparison to our study is difficult since Gölitz et al. [13] utilized different 2-D perfusion imaging technology and techniques.

There are limitations to the use of this 2-D perfusion technology in its current form. Currently, the DSA image obtained directly during the 2-D perfusion acquisition is acquired in a single plane rather than in 2 angiographic planes as typical with modern cerebral DSA. Moreover, the DSA image obtained directly from the 2-D perfusion acquisition is of lower quality than standard cerebral DSA, and thus administration of additional contrast for additional imaging may be required, prolonging overall procedure and anesthetic time. However, the only viable alternative methods for determining radiographic response of the microvasculature to vasospasm is CT or magnetic resonance perfusion imaging, which requires transfer of potentially intubated patients to alternative machines to acquire the data, greater imaging time, additional contrast dose, and potentially even higher levels of additional radiation with CT perfusion $[3,5,6,14]$. Additionally, metallic artefacts from coils or clips can complicate the interpretation of the results $[5,6,15]$. Current emerging tech- 
niques, such as cone beam CT perfusion software, are an alternative to 2-D perfusion software investigated in this study. The main difference between the 2-D perfusion software and the cone beam CT perfusion imaging is the additional higher dose acquisition for cone beam CT perfusion.

Our study was subjected to several limitations. Firstly, the small sample size in the analysis does not allow for a robust statistical assessment of the effect of treatment on parenchymal perfusion. Furthermore, there is the potential for overlap between vascular territories, which could influence the results due to the 2-D projection used to derive the data. The placement of the ROIs to assess the values of AT, MTT, and TTP was chosen by the software user, which could introduce selection bias. A larger prospective study is warranted to validate 2-D perfusion imaging technology as a means of evaluating radiological effectiveness of vasospasm management. As the technology improves, perfusion imaging may be able to investigate the role of constriction of the microvasculature in the pathology of vasospasm and to assess the clinical and radiological responses to vasospasm therapy. Additional limitations of this study include the assessment of a varied, operator-dependent dosage of intra-arterial vasodilator agents. Further investigation into single compared with multiple agents and also comparison of dosage range with this technique would be of use. Cardiac output can vary with the use of vasopressor agents, especially during intra-arterial vasodilator treatment; however, there is no current literature to quantify this. While this may be a limitation, our total acquisition time for this procedure always included contrast wash-in and wash-out time, and recording was performed until opacification of the major draining sinuses. This ensured that all patients had a recording time reflective of tissue perfusion.

Future studies directly comparing methods such as CT perfusion, cone beam CT perfusion, and magnetic resonance perfusion with real-time 2-D perfusion imaging would be of use in the further validation of this technique.

The technique described could potentially be semiautomated since ROI circles could be automatically inputted into the software. However, this would require some operator input to ensure that large vessels were avoided in the analysis to give an accurate representation of tissue perfusion, as performed in our study.

The technique described provides an objective measure of radiographic perfusion response to vasodilator treatment. Future uses for this technique could potentially be providing a guide to duration and dosage of treatments based on individual perfusion parameters during treatments. This would allow the customization of vasospasm treatment for each individual based on his or her own response to treatment. Similarly, if a "normal" perfusion parameter standard was established, this could be used as the bench mark for the termination of vasospasm treatment.

\section{Conclusion}

Real-time 2-D parenchymal perfusion imaging during endovascular vasospasm treatment procedures is feasible and provides a reliable semiquantitative measurement of angiographic treatment response.

\section{Disclosure Statement}

The authors declare that they have no financial or other conflicts of interest in relation to this research and its publication. 


\section{Author Contributions}

R.V.C. was responsible for project conception. L.-A.S., W.C., and R.V.C. were responsible for data acquisition and interpretation. C.D. and L.T.L. were responsible for data analysis. C.D. and A.H.O. were responsible for manuscript preparation. All authors were responsible for critical revisions of the manuscript. All authors gave final approval for the version to be published and agreed to be accountable for all aspects of the work in ensuring that questions related to the accuracy or integrity of any part of the work are appropriately investigated and resolved.

\section{References}

1 Ferguson S, Macdonald RL: Predictors of cerebral infarction in patients with aneurysmal subarachnoid hemorrhage. Neurosurgery 2007;60:658-667; discussion 667.

2 Rosengart AJ, Schultheiss KE, Tolentino J, Macdonald RL: Prognostic factors for outcome in patients with aneurysmal subarachnoid hemorrhage. Stroke 2007;38:2315-2321.

3 Findlay JM, Nisar J, Darsaut T: Cerebral vasospasm: a review. Can J Neurol Sci 2016;43:15-32.

4 Wan H, Loch Macdonald R: Circulatory and vascular changes after aneurysmal subarachnoid hemorrhage. J Neurosurg Sci 2011;55:329-341.

5 Baggott CD, Aagaard-Kienitz B: Cerebral vasospasm. Neurosurg Clin N Am 2014;25:497-528.

6 Levitt MR, Morton RP, Haynor DR, et al: Angiographic perfusion imaging: real-time assessment of endovascular treatment for cerebral vasospasm. J Neuroimaging 2014;24:387-392.

7 Strother C, Bender F, Deuerling-Zheng Y, et al: Parametric color coding of digital subtraction angiography. Am J Neuroradiol 2010;31:919-924.

8 Hirata M, Yoshimine T, Kato A, et al: Computational imaging of cerebral perfusion by real time processing of DSA images. Clinical applications. Neurol Res 1998;20:327-332.

9 Lin C, Hung S, Guo W, et al: Monitoring peri-therapeutic cerebral circulation time: a feasibility study using color-coded quantitative DSA in patients with steno-occlusive arterial disease. Am J Neuroradiol 2012;33: 1685-1690.

10 Konstas A, Goldmakher G, Lee T-Y, Lev M: Theoretic basis and technical implementations of CT perfusion in acute ischemic stroke, part 2: technical implementations. Am J Neuroradiol 2009;30:885-892.

11 Sanelli PC, Ugorec I, Johnson CE, et al: Using quantitative CT perfusion for evaluation of delayed cerebral ischemia following aneurysmal subarachnoid hemorrhage. Am J Neuroradiol 2011;32:2047-2053.

12 Ganguly A, Fieselmann A, Marks M, et al: Cerebral CT perfusion using an interventional C-arm imaging system: cerebral blood flow measurements. Am J Neuroradiol 2011;32:1525-1531.

13 Gölitz P, Kaschka I, Lang S, Roessler K, Knossalla F, Doerfler A: Real-time, in vivo monitoring, and quantitative assessment of intra-arterial vasospasm therapy. Neurocrit Care 2016;25:40-46.

14 Gelfand AA, Josephson SA: Substantial radiation exposure for patients with subarachnoid hemorrhage. J Stroke Cerebrovasc Dis 2011;20:131-133.

15 McGuinness B, Gandhi D: Endovascular management of cerebral vasospasm. Neurosurg Clin N Am 2010;21: 281-290. 